\title{
利用碳碳参键断裂重组反应合成 2,4,4-三氯慕-1(4H)-酮衍生物
}

\author{
蒋文婕 ${ }^{\dagger}, a, b$ 宋雅婷 ${ }^{\dagger}, a, b$ 韦晓静 ${ }^{\dagger}, a$ 徐 义 \\ 陆 娟 ${ }^{\dagger}, a$ 姜 波*,a,b 郝文娟 ${ }^{a}$ \\ ( ${ }^{a}$ 江苏师范大学化学与材料科学学院 徐州 221116) \\ ( ${ }^{b}$ 江苏师范大学敬文书院＼cjkstart徐州 221116)
}

\begin{abstract}
摘要 以炔烃-联烯酮为原料, 水作为亲核试剂, 通过 $N$-氯代丁二酰亚胺 $(\mathrm{NCS})$ 介导的 $[2+2]$ 环加成和碳碳参键断裂重 组反应合成了 18 个 2,4,4-三氯荎-1(4H)-酮衍生物, 产率为 $55 \%$ \% $88 \%$. 所得产物的结构经 NMR 和 IR 以及 HRMS 等表 征, 其中目标产物 $2 \mathrm{e}$ 的结构经 $\mathrm{X}$ 单晶分析确认. 该反应条件温和、操作简单、无需金属催化剂且产率良好, 为合成官 能化的 1-芸酮衍生物提供了一种新的高效的合成策略.
\end{abstract}

关键词＼cjkstart炔烃-联烯酮; [2+2]环加成; 碳碳参键断裂重组反应; 2,4,4-三氯菜-1 $(4 H)$-酮衍生物

\section{C-C Triple Bond Cleavage/Rearrangement Reaction for Accessing 2,4,4-Trichloronaphthalen-1(4H)-ones}

\author{
Jiang, Wenjie $\mathrm{i}^{\dagger, a, b} \quad$ Song, Yating ${ }^{\dagger, a, b} \quad$ Wei, Xiaojing ${ }^{\dagger, a} \quad \mathrm{Xu}, \mathrm{Yi}^{\dagger, a}$ \\ Lu, Juan ${ }^{\dagger, a}$ Jiang, Bo ${ }^{*, a, b} \quad$ Hao, Wenjuan $^{a}$ \\ ( ${ }^{a}$ School of Chemistry \& Materials Science, Jiangsu Normal University, Xuzhou 221116) \\ $\left({ }^{b}\right.$ C.W. Chu College, Jiangsu Normal University, Xuzhou 221116)
}

\begin{abstract}
N}$-Chlorosuccinimide (NCS)-mediated [2+2] cycloaddition and C-C triple bond cleavage/rearrangement reaction of yne-allenones and $\mathrm{H}_{2} \mathrm{O}$ as a nucleophilic reagent enabled the formation of 18 examples of 2,4,4-trichloronaphthalene$1(4 H)$-one derivatives with $55 \% \sim 88 \%$ yields. The structures of these obtainable products were based on their NMR, IR and HRMS data, among which the structure of product $2 \mathrm{e}$ was confirmed by X-ray analysis. This metal-free transformation features mild conditions, simple operation and high reaction yields. It provides an effective and new protocol for the synthesis of trichloro-substituted naphthalene-1(4H)-ones.

Keywords yne-allenones; $[2+2]$ cycloaddition; $\mathrm{C}-\mathrm{C}$ triple bond cleavage/rearrangement; 2,4,4-trichloronaphthalene$1(4 H)$-ones
\end{abstract}

近年来, 碳碳参键的断裂重组反应在有机合成中是 一类非常有用的合成策略，展现出简单高效、原子经济 等优点 ${ }^{[1]}$. 此反应可通过非传统的断裂方式, 对碳碳参 键进行重组, 合成了一些具有重要价值的分子结构单 元 $^{[2]}$. 早期, 对 $\mathrm{C} \equiv \mathrm{C}$ 键切割重组的研究主要集中在化 学计量的有机金属化合物参与的反应, 例如金属配合物 中炔烃与配体的断裂 ${ }^{[3]}$ 和氧化裂解 ${ }^{[4]}$, 该方法需要大量 的有机金属化合物, 且适用范围较窄. 此后, 化学工作
者们发展了过渡金属催化的碳碳参键的断裂和重组反 应，分别使用催化量的银 ${ }^{[5]} 、 \mathrm{c}^{[6]}$ 、铑 ${ }^{[7]} 、$ 金 $^{[8]}$ 、铜 ${ }^{[9]}$ 、 钓 ${ }^{[10]}$ 等金属盐为催化剂, 有效地实现了 $\mathrm{C} \equiv \mathrm{C}$ 键的切割 和重组，构筑了一系列结构不同的有机分子骨架. 然而 这些方法都使用了价格昂贵的金属催化剂，易于金属残 留, 不利于所合成产物的药物篮选 ${ }^{[11]}$. 因此, 发展一种 绿色、高效且原子经济的合成方法来实现无金属催化的 $\mathrm{C} \equiv \mathrm{C}$ 键的切割重组反应是非常值得研究的课题.

\footnotetext{
* Corresponding author. E-mail: jiangchem@jsnu.edu.cn

Received November 10, 2018; revised December 7, 2018; published online December 21, 2018

Project supported by the National Natural Science Foundation of China (No. 21602087), the Top-notch Academic Programs Project of Jiangsu Higher Education Institutions, the Natural Science Foundation of Jiangsu Province (No. BK20160212), and the Qing Lan Project of Jiangsu Education Committee. 国家自然科学基金(No. 21602087)、江苏高校品牌专业建设工程基金、江苏省自然科技基金(No. BK20160212)和江苏省青蓝工程资助项目.

共同第一作者(These authors contributed equally to this work)
} 
一直以来我们课题组在串联环化反应方面积累了 丰富的经验, 合成了大量具有生物重要性的碳环和杂环 分子 ${ }^{[12]}$, 且在利用 $\mathrm{C} \equiv \mathrm{C}$ 键的切割重组方面也取得了一 些进展 ${ }^{[13]}$. 如使用炔烃-联烯酮与 1.5 equiv.的 $N$-氯代丁 二酰亚胺(NCS)为原料, 水作为亲核试剂, 合成了一系

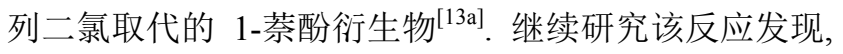
使用过量 NCS (3.0 equiv.) 与炔烃-联烯酮衍生物和水进 行多组分串联反应时, 没有得到预期的二氯代 1 -萗酚衍 生物, 而是高效地产生了一类结构新颖的三氯代荎-1酮衍生物, 实现了同碳原子的双氯代反应(Scheme 1).

Our previous work

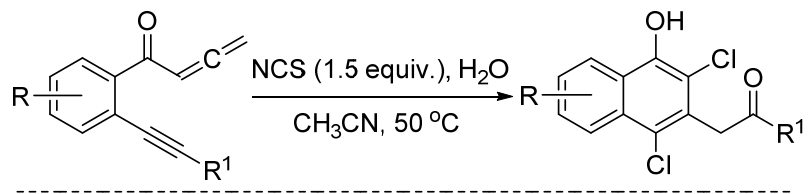

This work

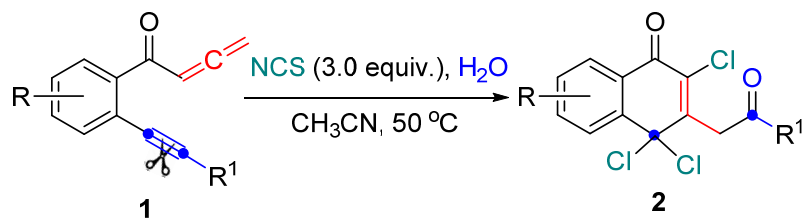

图式 1 三氯代菜-1-酮衍生物 2 的合成

Scheme 1 Synthesis of trichloro-substituted naphthalene-1(4H)ones 2

\section{1 结果与讨论}

\section{1 反应条件的优化}

反应开始以炔烃-联烯酮 (1a)、NCS 与水为起始原 料, 研究不同溶剂、不同温度和不同投料比及反应环境 对三氯代荎-1-酮产物产率的影响. 实验结果见表 1. 利 用炔烃-联烯酮 1a、3 equiv. NCS 与 1.5 equiv.水在 $50{ }^{\circ} \mathrm{C}$ 下进行反应, 篮选了不同溶剂, 如乙腈 $(\mathrm{MeCN}) 、 1,2-二$ 氯乙烷 $(\mathrm{DCE})$ 、甲醇 $\left(\mathrm{CH}_{3} \mathrm{OH}\right) 、 1,4$-二氧六环 $(1,4$-dioxane)、四氢呋喃(THF)、二氯甲烷(DCM) 和 $N, N$-二甲基 甲酰胺(DMF). 在乙腈中反应最好, 产率达到 $66 \%$, 在 DCE、1,4-二氧六环和 DCM 中产物的产率有较大幅度 降低，在甲醇和 THF 中反应效果不好，几乎检测不到产 物, 在 DMF 中产率中等, 为 $48 \%$ (表 1 中 Entries 1 7). 接着考察不同温度对反应的影响. 升高或降低反应温度 都使反应的产率有所降低(表 1, Entry 1 vs. Entries 8 10). 随后对反应投料比进行篮选(表 1, Entries 11 13), 发现炔烃-联烯酮与 NCS 投料比为 $1: 3$ 时, 反应效果最 优, 给出目标产物的产率为 $66 \%$ (表 1, Entry 1). 最后对 反应环境进行了调整. 氩气条件下也没有提高反应的产 率(表 1 中 Entry 14), 鉴于氩气条件的复杂操作, 该反应 仍在空气环境下实施. 综上所述, 优化的反应条件是:
乙腈为反应溶剂, 溶剂用量 $2.0 \mathrm{~mL}$, 炔烃-联烯酮与 $\mathrm{NCS}$ 的物质的量之比为 $1: 3$, 水为 1.5 equiv., 反应温度 为 $50{ }^{\circ} \mathrm{C}$.

表 1 化合物 $2 \mathrm{a}$ 反应条件的优化 ${ }^{a}$

Table 1 Optimization of reaction conditions for the synthesis of $\mathbf{2 a}^{a}$

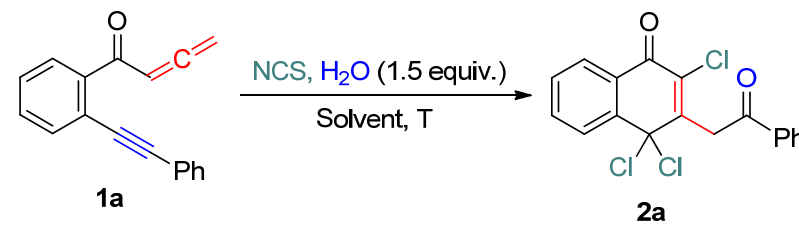

\begin{tabular}{|c|c|c|c|c|}
\hline Entry & $\begin{array}{c}\text { 1a: NCS } \\
\text { (mole ratio) }\end{array}$ & Solvent & $T /{ }^{\circ} \mathrm{C}$ & Yield $^{b} / \%$ \\
\hline 1 & $1: 3$ & $\mathrm{CH}_{3} \mathrm{CN}$ & 50 & 66 \\
\hline 2 & $1: 3$ & DCE & 50 & 28 \\
\hline 3 & $1: 3$ & $\mathrm{CH}_{3} \mathrm{OH}$ & 50 & N.D. ${ }^{c}$ \\
\hline 4 & $1: 3$ & 1,4-Dioxane & 50 & 25 \\
\hline 5 & $1: 3$ & THF & 50 & Trace \\
\hline 6 & $1: 3$ & DCM & 50 & 30 \\
\hline 7 & $1: 3$ & DMF & 50 & 48 \\
\hline 8 & $1: 3$ & $\mathrm{CH}_{3} \mathrm{CN}$ & 60 & 59 \\
\hline 9 & $1: 3$ & $\mathrm{CH}_{3} \mathrm{CN}$ & 40 & 63 \\
\hline 10 & $1: 3$ & $\mathrm{CH}_{3} \mathrm{CN}$ & 30 & 55 \\
\hline 11 & $1: 2$ & $\mathrm{CH}_{3} \mathrm{CN}$ & 50 & 35 \\
\hline 12 & $1: 3.5$ & $\mathrm{CH}_{3} \mathrm{CN}$ & 50 & 58 \\
\hline 13 & $1: 4$ & $\mathrm{CH}_{3} \mathrm{CN}$ & 50 & 52 \\
\hline $14^{d}$ & $1: 3$ & $\mathrm{CH}_{3} \mathrm{CN}$ & 50 & 58 \\
\hline
\end{tabular}

${ }^{a}$ Reaction condition: 1a $(0.2 \mathrm{mmol}), \mathrm{NCS}(0.6 \mathrm{mmol}), \mathrm{H}_{2} \mathrm{O}$ (1.5 equiv.), solvent $(2.0 \mathrm{~mL})$, air conditions. ${ }^{b}$ Isolated yield based on substrate 1a. ${ }^{c}$ No detected (N. D.). ${ }^{d}$ Argon gas.

\section{2 反应底物的拓展}

在最佳优化条件下，对该反应体系的底物普适性进 行了探索(表 2$)$. 最初考察了连接在炔烃上的芳基 $\left(\mathrm{R}^{1}\right)$ 的 适用范围. 结构表明: 芳环 $\mathrm{R}^{1}$ 上的各种取代基均能兼容 此反应，如氟(1b)、氯(1c,1d)、溴(1e)、甲基(1f, 1g)、乙 基(1h)以及叔丁基(1i)，且能提供相应的目标产物 $\mathbf{2 b} \sim$ $2 \mathbf{i}$, 产率为 $55 \% \sim 88 \%$. 其中，与邻位和间位相比，在芳 环对位的同类取代基能提供更高产率的目标产物(2c vs $\mathbf{2 d} ; \mathbf{2 f} v s \mathbf{2 g}$ ). 此外，当炔基上的取代基 $\mathrm{R}^{1}$ 为萗基时，该 反应也能进行, 得到相应目标产物 $\mathbf{2 j}$, 产率为 $64 \%$. 随 后评估了炔烃-联烯酮的内芳环上电子效应对反应的影 响. 因此, 在芳环上的 4 或 5 位引入了不同电子性质的

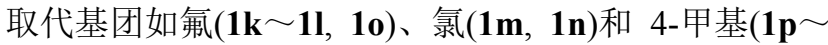
1r). 在相同的反应条件下，带有上述官能团的炔烃-联 烯酮均可顺利参与此反应，并以较高产率得到相应产物 $2 \mathbf{k} \sim 2 \mathbf{r}$, 产率为 $59 \% \sim 84 \%$. 总之，此类用于直接构建 三氯代荎-1-酮骨架的串联环加成策略是一类新颖可靠 的合成方法. 所合成的三氯代䒺-1-酮衍生物的结构均 
经 NMR 谱和 HR-MS 表征. 此外, 通过单晶 X 射线衍射 进一步确定了化合物 $\mathbf{2 e}$ 的结构, (图 1), CCDC 号为 1554753.

表 2 三氯代 1-䒺酮衍生物 $\mathbf{2} \mathbf{a} \sim \mathbf{2} \mathbf{r}$ 的合成

Table 2 Synthesis of trichloro-substituted naphthalene-1(4H)ones $\mathbf{2 a} \sim \mathbf{2 r}$<smiles>[R]C#Cc1ccccc1C(=O)C=C=C</smiles>

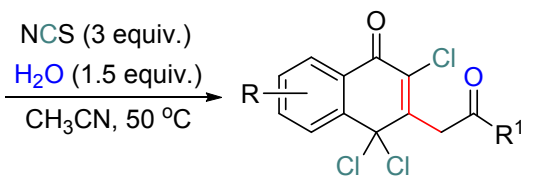
1 2<smiles>[R2]C(=O)CC1=C(Cl)C(=O)c2ccccc2C1(Cl)Cl</smiles>
2a $\sim 2 \mathbf{j}$<smiles>[R]C(=O)CC1=C(Cl)C(=O)c2cc(F)ccc2C1(Cl)Cl</smiles><smiles>O=C(CC1=C(Cl)C(=O)c2cc(Cl)ccc2C1(Cl)Cl)c1ccccc1</smiles>
$2 \mathrm{~m}(70 \%)$

2k, $\mathrm{R}^{1}=\mathrm{Ph}(65 \%)$ 2I, $\mathrm{R}^{1}=p$-Tolyl $(76 \%)$<smiles>O=C(CC1=C(Cl)C(=O)c2ccc(F)cc2C1(Cl)Cl)c1ccccc1</smiles><smiles></smiles>

$20(84 \%)$<smiles>[R]C(=O)CC1=C(Cl)C(=O)c2ccc(C)cc2C1(Cl)Cl</smiles>

$2 \mathrm{p} \mathrm{R} \mathrm{R}^{1}=\mathrm{Ph}(60 \%)$ $2 q R^{1}=4-\mathrm{ClC}_{6} \mathrm{H}_{4}(65 \%)$ 2r R $\mathrm{R}^{1}=p$-Tolyl (59\%) $2 p \sim 2 r$

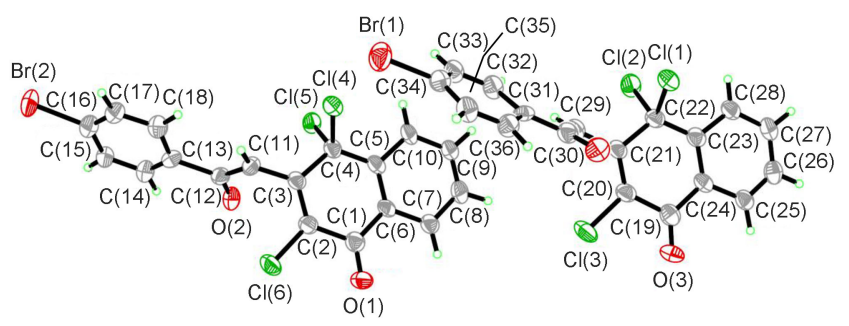

图 1 三氯代萗-1-酮衍生物 $2 \mathrm{e}$ 的晶体结构图

Figure 1 X-Ray structure of trichloro-substituted naphthalene1(4H)-one $2 \mathbf{e}$

\section{3 反应机理探讨}

为了探究反应机理, 我们实施了相关的控制反应, 将已制备的中间体 Int-3 与 1 equiv. 的 $N$-氯代丁二酰亚胺 (NCS) 在标准条件下进行了尝试. 非常幸运的是, 此反 应能顺利进行, 得到相应的产物 2a, 收率为 70\% (Eq. 1). 由此可推断该反应过程中可能涉及中间体 Int-3 的形

成.<smiles>[CH2-][PH2+]C(=O)Cc1c(Cl)c(O)c2ccccc2c1Cl</smiles>

Int-3<smiles>O=C(CC1=C(Cl)C(=O)c2ccccc2C1(Cl)Cl)c1ccccc1</smiles>

根据文献报道 ${ }^{[14]}$ 和上述的实验结果，提出一个可 能的反应机理. 如 Scheme 2 所示, 首先炔烃-联烯酮 1 发生分子内的 $[2+2]$ 环加成 $^{[15]}$, 生成环丁烯中间体 $\mathbf{A}$ (已被理论计算证实 $)^{[13 \mathrm{c}]}$. 随后中间体 $\mathbf{A}$ 在 $N$-氯代丁二 酰亚胺和水的存在下，通过卤代羟基化得到中间体 $\mathbf{C}$, 再经历环丁烯开环反应和质子转移(P.T.)形成中间体 $\mathbf{D}$. 最后，中间体 $\mathbf{D}$ 与 $N$-氯代丁二酰亚胺经过连续的两次 氯代反应得到最终产物 $\mathbf{2}$.<smiles>[R]C#Cc1ccccc1C(=O)C=C=C</smiles>

B<smiles>[R]C(=O)Cc1c(CC[Al])c(O)c2ccccc2c1Cl</smiles>

D<smiles>[R]C(=O)Cc1c(Cl)c(Cl)c2ccccc2c1O</smiles><smiles>[R]C(=O)CC1=C(Cl)C(=O)c2ccccc2C1(Cl)Cl</smiles><smiles></smiles>

图式 2 生成化合物 2 的可能机理

Scheme 2 Proposed mechanism for the synthesis of products 2

\section{2 结论}

在无金属催化条件下, 将炔烃-联烯酮与 $N$-氯代丁 二酰亚胺和水进行多组分反应, 经历 $[2+2]$ 环加成、环 
丁烯原位开环及三次氯化反应, 高效地合成了 18 例 2,4,4-三氯䒺-1(4H)-酮衍生物，产率为 $55 \% \sim 88 \%$. 该反 应实现了温和条件下的碳碳参键的切割和重组, 完成了 2,4,4-三氯芸-1(4H)-酮骨架的有效构筑, 为合成多官能 化的荎䣶衍生物提供了一种有效新颖的方法. 此外, 该 串联反应还具有反应温和、环境友好、原子经济、操作 简单等优点.

\section{3 实验部分}

\section{1 仪器与试剂}

仪器: 熔点测定仪(XT-5 型); 红外光谱仪(FTIRTensor 27 型); 质谱仪(Bruker microTOF-Q II 型); 核磁 共振仪(Bruker DPX $400 \mathrm{MHz}$ 型); 微波合成仪(瑞典 Biotage); 上海暗箱紫外分析仪(ZF-20D 型); 四圆衍射 仪(Siemens P4 型)等.

试剂: DMSO- $d_{6}$ (内标为 $\mathrm{TMS}$ )、 $\mathrm{CDCl}_{3}$ (内标为 TMS)、乙酸乙酯(分析纯)、石油醚(分析纯)、乙腈(分析 纯)、 $N$-氯代丁二酰亚胺等.

\section{2 实验方法}

将炔烃-联烯酮 $(0.2 \mathrm{mmol}) 、 N$-氯代丁二酰亚胺 $(0.6$ $\mathrm{mmol})$ 、水(1.5 equiv。)以及乙腈 $2.0 \mathrm{~mL}$ 依次加入到 10 $\mathrm{mL}$ 反应瓶中, 密封加热至 $50{ }^{\circ} \mathrm{C}$, 采用薄层色谱(TLC) 跟踪监测直至反应完全, 使用 200 300 目的硅胶, 乙酸 乙酯和石油醚混合物 $(V: V=1: 30)$ 作为洗脱剂, 柱层 析后旋蒸得到一系列纯净的白色固体产物 $\mathbf{2 a} \sim \mathbf{2 r}$.

2,4,4-三氯-3-(2-氧代-2-苯乙基)䒬-1(4H)-酮(2a): 白 色固体, 产率 66\%. m.p. $174 \sim 176{ }^{\circ} \mathrm{C} ;{ }^{1} \mathrm{H}$ NMR (400 MHz, DMSO- $\left.d_{6}\right) \delta: 8.23(\mathrm{~d}, J=7.6 \mathrm{~Hz}, 1 \mathrm{H}, \mathrm{ArH}), 8.19 \sim$ 8.17 (m, 2H, ArH), 8.11 8.11 (m, 1H, ArH), 8.01 7.96 $(\mathrm{m}, 1 \mathrm{H}, \mathrm{ArH}), 7.76 \sim 7.74(\mathrm{~m}, 2 \mathrm{H}, \mathrm{ArH}), 7.64 \sim 7.60(\mathrm{~m}$, $2 \mathrm{H}, \mathrm{ArH}), 4.86\left(\mathrm{~s}, 2 \mathrm{H}, \mathrm{CH}_{2}\right) ;{ }^{13} \mathrm{C} \mathrm{NMR}(100 \mathrm{MHz}$, DMSO- $\left.d_{6}\right) \delta: 192.3,175.2,149.5,146.9,142.3,136.1$, $135.8,134.4,131.7,130.0,129.5,128.8,128.3,126.8$, 125.4, 123.3, 82.7, 43.6; IR (KBr) v: 2992, 1697, 1674, 1448, 1318, 1269, 1214, 974, 849, 766, $711 \mathrm{~cm}^{-1}$. HRMS (APCI-TOF) calcd for $\mathrm{C}_{18} \mathrm{H}_{12} \mathrm{Cl}_{3} \mathrm{O}_{2}[\mathrm{M}+\mathrm{H}]^{+} 364.9903$; found 364.9919 .

2,4,4-三氯-3-[2-(4-氟苯基)-2-氧代乙基]菜-1(4H)-酮 (2b): 白色固体, 产率 55\%. m.p. $151 \sim 153{ }^{\circ} \mathrm{C}$; ${ }^{1} \mathrm{H} \mathrm{NMR}$ $\left(400 \mathrm{MHz}, \mathrm{DMSO}-d_{6}\right) \delta: 8.29 \sim 8.23(\mathrm{~m}, 3 \mathrm{H}, \mathrm{ArH}), 8.11 \sim$ $8.10(\mathrm{~m}, 1 \mathrm{H}, \mathrm{ArH}), 8.00 \sim 7.97(\mathrm{~m}, 1 \mathrm{H}, \mathrm{ArH}), 7.79 \sim 7.75$ (m, 1H, ArH), 7.47 7.43 (m, 2H, ArH), 4.85 (s, 2H, $\mathrm{CH}_{2}$ ); ${ }^{13} \mathrm{C}$ NMR (100 MHz, DMSO- $\left.d_{6}\right) \delta$ : 191.1, 175.2, 165.9 $\left({ }^{1} J_{\mathrm{CF}}=251.4 \mathrm{~Hz}\right), 146.8,142.3,135.8,134.3,132.8\left({ }^{4} J_{\mathrm{CF}}=\right.$ $2.8 \mathrm{~Hz}), 132.0\left({ }^{3} J_{\mathrm{CF}}=9.6 \mathrm{~Hz}\right), 131.7,130.1,126.8,125.4$, $116.5\left({ }^{2} J_{\mathrm{CF}}=21.8 \mathrm{~Hz}\right), 82.7,43.5$; IR (KBr) v: 3020, 1691, $1672,1555,1337,1289,1170,952,837,726,717 \mathrm{~cm}^{-1}$. HRMS (APCI-TOF) calcd for $\mathrm{C}_{18} \mathrm{H}_{11} \mathrm{Cl}_{3} \mathrm{FO}_{2}[\mathrm{M}+\mathrm{H}]^{+}$ 382.9809; found 382.9817 .

2,4,4-三氯-3-[2-(4-氯苯基)-2-氧代乙基]菜-1(4H)-酮 (2c): 白色固体，产率 78\%. m.p. $170 \sim 172{ }^{\circ} \mathrm{C} ;{ }^{1} \mathrm{H}$ NMR $\left(400 \mathrm{MHz}, \mathrm{DMSO}-d_{6}\right) \delta: 8.25 \sim 8.19(\mathrm{~m}, 3 \mathrm{H}, \mathrm{ArH}), 8.11 \sim$ 8.10 (m, 1H, ArH), 7.99 (s, 1H, ArH), 7.77 (s, 1H, ArH), $7.71 \sim 7.68(\mathrm{~m}, 2 \mathrm{H}, \mathrm{ArH}), 4.86\left(\mathrm{~s}, 2 \mathrm{H}, \mathrm{CH}_{2}\right) ;{ }^{13} \mathrm{C} \mathrm{NMR}$ $\left(100 \mathrm{MHz}, \mathrm{DMSO}-d_{6}\right) \delta: 191.6,175.2,149.5,146.7,142.3$, $139.4,138.3,135.8,134.8,130.8,130.2,129.6,129.1$, 126.8, 125.4, 123.3, 82.7, 43.5; IR (KBr) v: 3018, 1697, $1675,1507,1320,1276,1094,974,865,712,692 \mathrm{~cm}^{-1}$. HRMS (APCI-TOF) calcd for $\mathrm{C}_{18} \mathrm{H}_{11} \mathrm{Cl}_{4} \mathrm{O}_{2}[\mathrm{M}+\mathrm{H}]^{+}$ 398.9513; found 398.9527.

2,4,4-三氯-3-[2-(2-氯苯基)-2-氧乙基]菜-1(4H)-酮 (2d): 白色固体，产率 $72 \%$. m.p. 161 $163{ }^{\circ} \mathrm{C} ;{ }^{1} \mathrm{H}$ NMR $\left(400 \mathrm{MHz}, \mathrm{DMSO}-d_{6}\right) \delta: 8.24(\mathrm{~d}, J=8.0 \mathrm{~Hz}, 1 \mathrm{H}, \mathrm{ArH})$, $8.11 \sim 8.09(\mathrm{~m}, 1 \mathrm{H}, \mathrm{ArH}), 8.01 \sim 7.93(\mathrm{~m}, 2 \mathrm{H}, \mathrm{ArH})$, $7.79 \sim 7.75(\mathrm{~m}, 1 \mathrm{H}, \mathrm{ArH}), 7.64 \sim 7.62(\mathrm{~m}, 2 \mathrm{H}, \mathrm{ArH})$, $7.58 \sim 7.55(\mathrm{~m}, 1 \mathrm{H}, \mathrm{ArH}), 4.77\left(\mathrm{~s}, 2 \mathrm{H}, \mathrm{CH}_{2}\right) ;{ }^{13} \mathrm{C} \mathrm{NMR}$ $\left(100 \mathrm{MHz}, \mathrm{DMSO}-d_{6}\right) \delta: 194.2,175.2,145.7,142.1,137.9$, $135.9,134.6,133.5,131.8,131.3,130.4,130.0,128.2$, 126.8, 125.4, 82.5, 46.8; IR (KBr) v: 3036, 1680, 1665, 1410, 1220, 1126, 1004, 954, 890, 792, $682 \mathrm{~cm}^{-1}$. HRMS (APCI-TOF) calcd for $\mathrm{C}_{18} \mathrm{H}_{11} \mathrm{Cl}_{4} \mathrm{O}_{2}[\mathrm{M}+\mathrm{H}]^{+}$398.9514; found 398.9534 .

3-[2-(4-溴苯基)-2-氧代乙基]-2,4,4-三氯萘-1(4H)-酮 (2e): 白色固体, 产率 $88 \%$. m.p. $165 \sim 167{ }^{\circ} \mathrm{C} ;{ }^{1} \mathrm{H}$ NMR $\left(400 \mathrm{MHz}, \mathrm{DMSO}-d_{6}\right) \delta: 8.23(\mathrm{~d}, J=8.0 \mathrm{~Hz}, 1 \mathrm{H}, \mathrm{ArH})$, $8.13 \sim 8.10$ (m, 3H, ArH), 7.99 (s, 1H, ArH), 7.84 (d, J= $8.4 \mathrm{~Hz}, 2 \mathrm{H}, \mathrm{ArH}), 7.77$ (s, 1H, ArH), $4.85\left(\mathrm{~s}, 2 \mathrm{H}, \mathrm{CH}_{2}\right) ;{ }^{13} \mathrm{C}$ NMR $\left(100 \mathrm{MHz}, \mathrm{DMSO}-d_{6}\right) \delta: 191.8,175.2,149.5,146.7$, $142.3,135.8,132.5,132.0,130.9,130.3,130.1,129.4$, 128.7, 126.8, 125.4, 123.3, 82.7, 43.5; IR (KBr) v: 3026, $1698,1675,1585,1397,1319,1270,972,818,725,710$ $\mathrm{cm}^{-1}$. HRMS (APCI-TOF) calcd for $\mathrm{C}_{18} \mathrm{H}_{11} \mathrm{BrCl}_{3} \mathrm{O}_{2}[\mathrm{M}+$ $\mathrm{H}]^{+}$442.9008; found 442.9024 .

2,4,4-三氯-3-[2-氧代-2-(对甲苯基)乙基]菜-1-(4H)酮(2f): 白色固体，产率 $82 \%$. m.p. $168 \sim 170{ }^{\circ} \mathrm{C} ;{ }^{1} \mathrm{H}$ NMR (400 MHz, DMSO-d $\left.d_{6}\right) \delta: 8.23(\mathrm{~d}, J=8.4 \mathrm{~Hz}, 1 \mathrm{H}$, $\operatorname{ArH}), 8.10$ (d, $J=8.4 \mathrm{~Hz}, 1 \mathrm{H}, \mathrm{ArH}), 8.07$ (d, $J=8.4 \mathrm{~Hz}$, $2 \mathrm{H}, \mathrm{ArH}), 8.00 \sim 7.96(\mathrm{~m}, 1 \mathrm{H}, \mathrm{ArH}), 7.78 \sim 7.75(\mathrm{~m}, 1 \mathrm{H}$, $\operatorname{ArH}), 7.42$ (d, J=8.0 Hz, 2H, ArH), 4.81 (s, 2H, $\mathrm{CH}_{2}$ ), $2.43\left(\mathrm{~s}, 3 \mathrm{H}, \mathrm{CH}_{3}\right) ;{ }^{13} \mathrm{C}$ NMR $\left(100 \mathrm{MHz}, \mathrm{DMSO}-d_{6}\right) \delta$ : 
$191.8,175.2,149.4,147.1,145.0,142.3,135.8,133.7$, $131.7,130.0,129.4,129.0,128.4,126.8,125.4,123.3$, 82.7, 43.4, 21.7; IR (KBr) v: 3086, 1694, 1678, 1580, 1411, 1272, 1152, 980, 788, 706, $692 \mathrm{~cm}^{-1}$. HRMS (APCI-TOF) calcd for $\mathrm{C}_{19} \mathrm{H}_{14} \mathrm{Cl}_{3} \mathrm{O}_{2}[\mathrm{M}+\mathrm{H}]^{+}$379.0059; found 379.0071 .

2,4,4-三氯-3-[2-氧代-2-(间甲苯基)乙基]萗-1-(4H)酮(2g): 白色固体, 产率 $61 \%$. m.p. $154 \sim 156{ }^{\circ} \mathrm{C} ;{ }^{1} \mathrm{H}$ NMR $\left(400 \mathrm{MHz}\right.$, DMSO- $\left.d_{6}\right) \delta: 8.22(\mathrm{~d}, J=7.2 \mathrm{~Hz}, 1 \mathrm{H}$, $\mathrm{ArH}), 8.11 \sim 8.09(\mathrm{~m}, 1 \mathrm{H}, \mathrm{ArH}), 8.00 \sim 7.95(\mathrm{~m}, 3 \mathrm{H}, \mathrm{ArH})$, $7.78 \sim 7.74(\mathrm{~m}, 1 \mathrm{H}, \mathrm{ArH}), 7.56 \sim 7.48(\mathrm{~m}, 2 \mathrm{H}, \mathrm{ArH}), 4.83$ (s, $\left.2 \mathrm{H}, \mathrm{CH}_{2}\right), 2.43\left(\mathrm{~s}, 3 \mathrm{H}, \mathrm{CH}_{3}\right) ;{ }^{13} \mathrm{C}$ NMR $(100 \mathrm{MHz}$, DMSO- $\left.d_{6}\right) \delta: 192.3,175.2,149.5,147.0,142.3,138.9$, $138.3,136.1,135.8,131.7,130.0,129.2,126.8,126.0$, $125.4,123.3,82.7,43.6,21.3$; IR (KBr) v: 3031, 1694, 1674, 1583, 1400, 1324, 1184, 887, 853, 785, $707 \mathrm{~cm}^{-1}$. HRMS (APCI-TOF) calcd for $\mathrm{C}_{19} \mathrm{H}_{14} \mathrm{Cl}_{3} \mathrm{O}_{2}[\mathrm{M}+\mathrm{H}]^{+}$ 379.0059; found 379.0077.

2,4,4-三氯-3-[2-(4-乙基苯基)-2-氧乙基]萗-1(4H)-酮 (2h): 白色固体, 产率 80\%. m.p. $149 \sim 151{ }^{\circ} \mathrm{C} ;{ }^{1} \mathrm{H}$ NMR (400 MHz, DMSO- $\left.d_{6}\right) \delta: 8.23(\mathrm{~d}, J=8.0 \mathrm{~Hz}, 1 \mathrm{H}, \mathrm{ArH})$, $8.12(\mathrm{~d}, J=8.0 \mathrm{~Hz}, 3 \mathrm{H}, \mathrm{ArH}), 8.00 \sim 7.97(\mathrm{~m}, 1 \mathrm{H}, \mathrm{ArH})$, $7.78 \sim 7.75(\mathrm{~m}, 1 \mathrm{H}, \operatorname{ArH}), 7.45(\mathrm{~d}, J=8.0 \mathrm{~Hz}, 2 \mathrm{H}, \operatorname{ArH})$, $4.82\left(\mathrm{~s}, 2 \mathrm{H}, \mathrm{CH}_{2}\right), 2.73\left(\mathrm{~m}, 2 \mathrm{H}, \mathrm{CH}_{2}\right), 1.25 \sim 1.21(\mathrm{~m}, 3 \mathrm{H}$, $\left.\mathrm{CH}_{3}\right) ;{ }^{13} \mathrm{C}$ NMR (100 MHz, DMSO- $\left.d_{6}\right) \delta: 191.8,175.2$, $151.0,147.1,142.3,135.8,134.3,133.9,131.7,130.0$, 129.1, 128.8, 128.5, 126.8, 125.4, 82.7, 43.5, 28.7, 15.7; IR (KBr) $v$ : 3010, 1687, 1672, 1500, 1300, 1176, 1054, 984, $875,742,699 \mathrm{~cm}^{-1}$. HRMS (APCI-TOF) calcd for $\mathrm{C}_{20} \mathrm{H}_{16}$ $\mathrm{Cl}_{3} \mathrm{O}_{2}[\mathrm{M}+\mathrm{H}]^{+}$393.0216; found 393.0238.

3-[2-(4-叔丁基)苯基]-2-氧乙基-4,4-三氯萗-1(4H)酮(2i): 白色固体, 产率 $73 \%$. m.p. $158 \sim 160{ }^{\circ} \mathrm{C} ;{ }^{1} \mathrm{H}$ NMR $\left(400 \mathrm{MHz}\right.$, DMSO- $\left.d_{6}\right) \delta: 8.23(\mathrm{~d}, J=8.0 \mathrm{~Hz}, 1 \mathrm{H}$, $\mathrm{ArH}), 8.12 \sim 8.09(\mathrm{~m}, 3 \mathrm{H}, \mathrm{ArH}), 8.00 \sim 7.96(\mathrm{~m}, 1 \mathrm{H}, \mathrm{ArH})$, $7.78 \sim 7.74(\mathrm{~m}, 1 \mathrm{H}, \mathrm{ArH}), 7.62(\mathrm{~d}, J=8.4 \mathrm{~Hz}, 2 \mathrm{H}, \mathrm{ArH})$, $4.82\left(\mathrm{~s}, 2 \mathrm{H}, \mathrm{CH}_{2}\right), 1.34\left(\mathrm{~s}, 9 \mathrm{H}, \mathrm{C}_{4} \mathrm{H}_{9}\right) ;{ }^{13} \mathrm{C}$ NMR $(100$ MHz, DMSO- $\left.d_{6}\right) \delta: 191.8,175.2,157.6,147.1,142.3$, $135.8,134.3,133.6,131.7,130.1,128.8,126.8,126.3$, $125.4,82.7,43.5,35.4,31.2$; IR (KBr) v: 3016, 1689, $1675,1400,1240,1146,1034,974,895,782,690 \mathrm{~cm}^{-1}$. HRMS (APCI-TOF) calcd for $\mathrm{C}_{22} \mathrm{H}_{20} \mathrm{Cl}_{3} \mathrm{O}_{2}[\mathrm{M}+\mathrm{H}]^{+}$ 421.0529; found 421.0551.

2,4,4-氯-3-[(2-萗-1-基)-2-䒬氧乙基]-1(4H)-酮 (2j): 白色固体, 产率 $64 \%$. m.p. $158 \sim 160{ }^{\circ} \mathrm{C} ;{ }^{1} \mathrm{H}$ NMR (400 MHz, DMSO- $\left.d_{6}\right) \delta: 9.00(\mathrm{~s}, 1 \mathrm{H}, \mathrm{ArH}), 8.26 \sim 8.20(\mathrm{~m}, 2 \mathrm{H}$,
ArH), $8.14 \sim 8.07$ (m, 3H, ArH), $8.06(\mathrm{~d}, J=8.0 \mathrm{~Hz}, 1 \mathrm{H}$, ArH), $8.01 \sim 7.97(\mathrm{~m}, 1 \mathrm{H}, \mathrm{ArH}), 7.79 \sim 7.67(\mathrm{~m}, 3 \mathrm{H}, \mathrm{ArH})$, $5.00\left(\mathrm{~s}, 2 \mathrm{H}, \mathrm{CH}_{2}\right) ;{ }^{13} \mathrm{C}$ NMR $\left(100 \mathrm{MHz}\right.$, DMSO- $\left.d_{6}\right) \delta$ : $192.3,175.2,147.1,142.3,135.8,134.4,133.4,132.7$, $131.8,131.2,130.2,129.6,129.1,128.2,127.7,126.8$, $125.5,124.0,82.8,43.6$; IR (KBr) $v: 3030,1685,1675$, 1400, 1200, 1120, 1024, 984, 891, 796, $680 \mathrm{~cm}^{-1}$. HRMS (APCI-TOF) calcd for $\mathrm{C}_{22} \mathrm{H}_{14} \mathrm{Cl}_{3} \mathrm{O}_{2}[\mathrm{M}+\mathrm{H}]^{+}$415.0059; found 415.0070 .

2,4,4-三氯-7-氟-3-(2-氧代-2-苯基乙基)萗-1(4H)-酮 (2k): 白色固体, 产率 65\%. m.p. 149 151 ${ }^{\circ} \mathrm{C} ;{ }^{1} \mathrm{H}$ NMR $\left(400 \mathrm{MHz}\right.$, DMSO- $\left.d_{6}\right) \delta: 8.34 \sim 8.30(\mathrm{~m}, 1 \mathrm{H}, \mathrm{ArH}), 8.23 \sim$ $8.13(\mathrm{~m}, 2 \mathrm{H}, \mathrm{ArH}), 7.90 \sim 7.79$ (m, 2H, ArH), 7.76 7.73 (m, 1H, ArH), 7.64 7.60 (m, 2H, ArH), $4.86\left(\mathrm{~s}, 2 \mathrm{H}, \mathrm{CH}_{2}\right)$; ${ }^{13} \mathrm{C}$ NMR (100 MHz, DMSO- $\left.d_{6}\right) \delta$ : 192.3, 174.4, 164.4 $\left({ }^{1} J_{\mathrm{CF}}=249.9 \mathrm{~Hz}\right), 147.2,138.7,136.1,135.5,134.5,134.1$, $133.4\left({ }^{5} J_{\mathrm{CF}}=8.5 \mathrm{~Hz}\right), 132.0\left({ }^{6} J_{\mathrm{CF}}=2.8 \mathrm{~Hz}\right), 129.4\left({ }^{4} J_{\mathrm{CF}}=\right.$ $10.1 \mathrm{~Hz}), 128.9,128.3,123.4\left({ }^{2} J_{\mathrm{CF}}=23.2 \mathrm{~Hz}\right), 112.7$ $\left({ }^{3} J_{\mathrm{CF}}=21.7 \mathrm{~Hz}\right), 82.0,43.6$; IR (KBr) $v: 3022,1689,1679$, 1300, 1235, 1110, 1010, 934, 865, 796, $680 \mathrm{~cm}^{-1}$. HRMS (APCI-TOF) calcd for $\mathrm{C}_{18} \mathrm{H}_{11} \mathrm{Cl}_{3} \mathrm{FO}_{2}[\mathrm{M}+\mathrm{H}]^{+}$382.9809; found 382.9830 .

2,4,4-三氯-7-氟-3-[2-氧代-2-(对甲苯基)乙基]萗1(4H)-酮(2I): 白色固体, 产率 76\%. m.p. $153 \sim 155{ }^{\circ} \mathrm{C}$; ${ }^{1} \mathrm{H}$ NMR $\left(400 \mathrm{MHz}\right.$, DMSO- $\left.d_{6}\right) \delta: 8.33 \sim 8.29(\mathrm{~m}, 1 \mathrm{H}$, ArH), 8.07 (d, $J=8.4 \mathrm{~Hz}, 2 \mathrm{H}, \operatorname{ArH}), 7.88 \sim 7.78(\mathrm{~m}, 2 \mathrm{H}$, $\mathrm{ArH}), 7.42(\mathrm{~d}, J=8.0 \mathrm{~Hz}, 2 \mathrm{H}, \mathrm{ArH}), 4.81\left(\mathrm{~s}, 2 \mathrm{H}, \mathrm{CH}_{2}\right)$, $2.42\left(\mathrm{~s}, 3 \mathrm{H}, \mathrm{CH}_{3}\right) ;{ }^{13} \mathrm{C}$ NMR $\left(100 \mathrm{MHz}\right.$, DMSO- $\left.d_{6}\right) \delta$ : $191.7,174.4,161.8\left({ }^{1} J_{\mathrm{CF}}=233.5 \mathrm{~Hz}\right), 147.3,138.7\left({ }^{6} J_{\mathrm{CF}}=\right.$ $3.2 \mathrm{~Hz}), 134.1,133.4\left({ }^{4} J_{\mathrm{CF}}=8.7 \mathrm{~Hz}\right), 132.8,130.0,128.4$, $127.9\left({ }^{5} J_{\mathrm{CF}}=7.6 \mathrm{~Hz}\right), 123.5\left({ }^{3} J_{\mathrm{CF}}=23.1 \mathrm{~Hz}\right), 119.4,112.7$ $\left({ }^{2} J_{\mathrm{CF}}=23.5 \mathrm{~Hz}\right), 82.0,43.5,21.7$; IR (KBr) $v: 3002,1680$, $1670,1310,1215,1070,1005,964,855,776,660 \mathrm{~cm}^{-1}$. HRMS (APCI-TOF) calcd for $\mathrm{C}_{19} \mathrm{H}_{13} \mathrm{Cl}_{3} \mathrm{FO}_{2}[\mathrm{M}+\mathrm{H}]^{+}$ 396.9965; found 396.9987.

2,4,4,7-四氯-3-(2-氧代-2-苯基乙基)芸-1(4H)-酮 (2m): 白色固体, 产率 70\%. m.p. 166 168 ${ }^{\circ} \mathrm{C} ;{ }^{1} \mathrm{H}$ NMR $\left(400 \mathrm{MHz}, \mathrm{DMSO}-d_{6}\right) \delta: 8.25(\mathrm{~d}, J=9.6 \mathrm{~Hz}, 1 \mathrm{H}, \mathrm{ArH})$, $8.20 \sim 8.15(\mathrm{~m}, 2 \mathrm{H}, \operatorname{ArH}), 8.09 \sim 8.00(\mathrm{~m}, 2 \mathrm{H}, \operatorname{ArH})$, $7.76 \sim 7.72(\mathrm{~m}, 1 \mathrm{H}, \mathrm{ArH}), 7.64 \sim 7.60(\mathrm{~m}, 2 \mathrm{H}, \mathrm{ArH}), 4.86$ (s, $\left.2 \mathrm{H}, \mathrm{CH}_{2}\right) ;{ }^{13} \mathrm{C}$ NMR (100 MHz, DMSO- $\left.d_{6}\right) \delta: 192.3$, $174.2,147.1,136.5,135.5,134.5,133.6,132.3,129.5$, $128.9,128.3,127.1,126.0,122.1,81.9,43.5$; IR (KBr) v: 3008, 1687, 1677, 1407, 1300, 1216, 1044, 978, 885, 762, $672 \mathrm{~cm}^{-1}$. HRMS (APCI-TOF) calcd for $\mathrm{C}_{18} \mathrm{H}_{11} \mathrm{Cl}_{4} \mathrm{O}_{2}$ 
$[\mathrm{M}+\mathrm{H}]^{+}$398.9513; found 398.9517.

2,4,4,6-四氯-3-[2-氧代-2-(对甲苯基)乙基]萘-1(4H)酮(2n): 白色固体, 产率 $83 \%$. m.p. $163 \sim 165{ }^{\circ} \mathrm{C} ;{ }^{1} \mathrm{H}$ NMR (400 MHz, DMSO- $\left.d_{6}\right) \delta: 8.22(\mathrm{~d}, J=7.6 \mathrm{~Hz}, 1 \mathrm{H}$, ArH), 8.07 (d, $J=8.4 \mathrm{~Hz}, 2 \mathrm{H}, \mathrm{ArH}), 8.00 \sim 7.95(\mathrm{~m}, 1 \mathrm{H}$, $\operatorname{ArH}), 7.79 \sim 7.73(\mathrm{~m}, 1 \mathrm{H}, \mathrm{ArH}), 7.42(\mathrm{~d}, J=8.4 \mathrm{~Hz}, 2 \mathrm{H}$, ArH), 4.80 (s, 2H, $\mathrm{CH}_{2}$ ), 2.42 (s, 3H, $\left.\mathrm{CH}_{3}\right) ;{ }^{13} \mathrm{C}$ NMR (100 $\left.\mathrm{MHz}, \mathrm{DMSO}-d_{6}\right) \delta$ : 191.8, 175.2, 147.1, 145.0, 142.3, $135.8,133.7,131.72,130.0,129.0,126.8,125.4,123.2$, 82.7, 43.4, 21.7; IR (KBr) v: 3052, 1690, 1676, 1240, 1205, 1040, 1001, 954, 875, 796, $670 \mathrm{~cm}^{-1}$. HRMS (APCI-TOF) calcd for $\mathrm{C}_{19} \mathrm{H}_{13} \mathrm{Cl}_{4} \mathrm{O}_{2}[\mathrm{M}+\mathrm{H}]^{+}$414.9640; found 414.9660 .

\section{2,4,4-三氯-6-氟-3-(2-氧代-2-苯基乙基)菜-1(4H)-酮} (2o): 白色固体, 产率 84\%. m.p. $167 \sim 169{ }^{\circ} \mathrm{C}$; ${ }^{1} \mathrm{H}$ NMR $\left(400 \mathrm{MHz}, \mathrm{CDCl}_{3}\right) \delta: 8.24 \sim 8.20(\mathrm{~m}, 1 \mathrm{H}, \mathrm{ArH}), 8.13 \sim$ $8.08(\mathrm{~m}, 2 \mathrm{H}, \mathrm{ArH}), 7.82 \sim 7.79(\mathrm{~m}, 1 \mathrm{H}, \mathrm{ArH}), 7.68 \sim 7.63$ $(\mathrm{m}, 1 \mathrm{H}, \mathrm{ArH}), 7.58 \sim 7.54(\mathrm{~m}, 2 \mathrm{H}, \mathrm{ArH}), 7.31 \sim 7.36(\mathrm{~m}$, $1 \mathrm{H}, \mathrm{ArH}), 4.67$ (s, 2H, CH$) ;{ }^{13} \mathrm{C} \mathrm{NMR}\left(100 \mathrm{MHz}, \mathrm{CDCl}_{3}\right)$ $\delta: 191.2,174.2,166.0\left({ }^{1} J_{\mathrm{CF}}=256.0 \mathrm{~Hz}\right), 145.7,145.1$ $\left({ }^{5} J_{\mathrm{CF}}=9.3 \mathrm{~Hz}\right), 136.0,134.9,133.9,130.2\left({ }^{4} J_{\mathrm{CF}}=9.6 \mathrm{~Hz}\right)$, $128.9,128.3,122.6\left({ }^{6} J_{\mathrm{CF}}=2.9 \mathrm{~Hz}\right), 118.7\left({ }^{3} J_{\mathrm{CF}}=22.2 \mathrm{~Hz}\right)$, $116.2\left({ }^{2} J_{\mathrm{CF}}=24.8 \mathrm{~Hz}\right), 81.3$, 42.9; IR (KBr) v: 3006, 1693, 1679, 1549, 1415, 1280, 1208, 1090, 862, 754, $705 \mathrm{~cm}^{-1}$. HRMS (APCI-TOF) calcd for $\mathrm{C}_{18} \mathrm{H}_{11} \mathrm{Cl}_{3} \mathrm{FO}_{2}[\mathrm{M}+\mathrm{H}]^{+}$ 382.9809; found 382.9820 .

2,4,4-三氯-6-甲基-3-(2-氧代-2-苯基乙基)萘-1(4H)酮(2p): 白色固体, 产率 $60 \%$. m.p. $187 \sim 189{ }^{\circ} \mathrm{C} ;{ }^{1} \mathrm{H}$ NMR (400 MHz, DMSO- $\left.d_{6}\right) \delta: 8.19 \sim 8.16(\mathrm{~m}, 2 \mathrm{H}, \mathrm{ArH})$, $8.04(\mathrm{~s}, 1 \mathrm{H}, \mathrm{ArH}), 8.00(\mathrm{~d}, J=8.0 \mathrm{~Hz}, 1 \mathrm{H}, \mathrm{ArH}), 7.76 \sim$ $7.73(\mathrm{~m}, 1 \mathrm{H}, \mathrm{ArH}), 7.64 \sim 7.56(\mathrm{~m}, 3 \mathrm{H}, \mathrm{ArH}), 4.84(\mathrm{~s}, 2 \mathrm{H}$, $\left.\mathrm{CH}_{2}\right), 2.53\left(\mathrm{~s}, 3 \mathrm{H}, \mathrm{CH}_{3}\right) ;{ }^{13} \mathrm{C}$ NMR (100 MHz, DMSO- $\left.d_{6}\right)$ $\delta: 192.4,175.0,146.8,146.7,142.4,136.1,134.5,134.3$, $132.7,130.0,129.5,128.8,126.9,123.2,82.8,43.5,21.9$; IR (KBr) v: 3046, 1703, 1674, 1483, 1322, 1213, 1091, 921, 804, 782, $708 \mathrm{~cm}^{-1}$. HRMS (APCI-TOF) calcd for $\mathrm{C}_{19} \mathrm{H}_{14} \mathrm{Cl}_{3} \mathrm{O}_{2}[\mathrm{M}+\mathrm{H}]^{+}$379.0059; found 379.0068 .

2,4,4-三氯-3-[2-(4-氯苯基)-2-氧代乙基]-6-甲基萗1(4H)-酮(2q): 白色固体, 产率 65\%. m.p. 189 191 ${ }^{\circ} \mathrm{C}$; ${ }^{1} \mathrm{H}$ NMR (400 MHz, DMSO-d $\left.{ }_{6}\right) \delta: 8.21$ (s, 1H, ArH), 8.19 (s, 1H, ArH), 8.04 (s, 1H, ArH), 8.00 (d, $J=8.0 \mathrm{~Hz}, 1 \mathrm{H}$, ArH), 7.70 (s, 1H, ArH), 7.68 (s, 1H, ArH), 7.58 (d, J=8.4 $\mathrm{Hz}, 1 \mathrm{H}, \mathrm{ArH}), 4.84\left(\mathrm{~s}, 2 \mathrm{H}, \mathrm{CH}_{2}\right) ;{ }^{13} \mathrm{C}$ NMR $(100 \mathrm{MHz}$, DMSO- $\left.d_{6}\right) \delta$ : 191.6, 174.9, 146.8, 146.4, 142.3, 139.4, $134.8,134.4,132.7,130.8,130.0,129.6,126.9,123.2$,
82.7, 43.5; IR (KBr) v: 3092, 1697, 1643, 1401, 1308, 1090, 978, 821, 776, 720, $703 \mathrm{~cm}^{-1}$. HRMS (APCI-TOF) calcd for $\mathrm{C}_{19} \mathrm{H}_{13} \mathrm{Cl}_{4} \mathrm{O}_{2}[\mathrm{M}+\mathrm{H}]^{+}$412.9670; found 412.9679 .

2,4,4-三氯-6-甲基-3-[2-氧代-2-(对甲苯基)乙基]䒬1(4H)-酮(2r): 白色固体，产率 59\%. m.p. $182 \sim 183{ }^{\circ} \mathrm{C}$; ${ }^{1} \mathrm{H}$ NMR $\left(400 \mathrm{MHz}, \mathrm{DMSO}-d_{6}\right) \delta: 8.09 \sim 8.02(\mathrm{~m}, 3 \mathrm{H}$, ArH), 7.99 (d, J=8.0 Hz, 1H, ArH), 7.59 7.56 (m, 1H, $\operatorname{ArH}), 7.42$ (d, J=8.0 Hz, 2H, ArH), 4.79 (s, 2H, $\mathrm{CH}_{2}$ ), 2.53 (s, 3H, $\left.\mathrm{CH}_{3}\right), 2.43$ (s, 3H, $\left.\mathrm{CH}_{3}\right) ;{ }^{13} \mathrm{C}$ NMR (100 MHz, DMSO- $\left.d_{6}\right) \delta: 191.8,175.0,146.8,145.0,142.4,134.3$, $133.7,132.7,129.9,129.4,128.9,126.8,123.3,82.8,43.4$, 21.8, 21.7; HRMS (APCI-TOF) calcd for $\mathrm{C}_{20} \mathrm{H}_{16} \mathrm{Cl}_{3} \mathrm{O}_{2}$ $[\mathrm{M}+\mathrm{H}]^{+}$393.0216; found 393.0238.

辅助材料(Supporting Information) 部分化合物的 ${ }^{1} \mathrm{H}$ $\mathrm{NMR}$ 和 ${ }^{13} \mathrm{C} \mathrm{NMR}$ 图谱. 这些材料可以免费从本刊网站 (http://sioc-journal.cn/)上下载.

\section{References}

[1] (a) For reviews, see: Jennings, P. W.; Johnson, L. L. Chem. Rev. 1994, 94, 2241.

(b) Rybtchinski, B.; Milstein, D. Angew. Chem., Int. Ed. 1999, 38, 870 .

[2] (a) Jiang, Q.; Zhao, A.; Xu, B.; Jia, J.; Liu, X.; Guo, C. J. Org. Chem. 2014, 79, 2709

(b) Okamoto, N.; Ishikura, M.; Yanada, R. Org. Lett. 2013, 15, 2571 .

[3] (a) Chamberlin, R. L. M.; Rosenfeld, D. C.; Wolczanski, P. T.; Lobkovsky, E. B. Organometallics 2002, 21, 2724.

(b) Adams, H.; Guio, L. V. Y.; Morris, M. J.; Spey, S. E. J. Chem. Soc., Dalton Trans. 2002, 2907.

(c) O'Connor, J. M.; Pu, L. J. Am. Chem. Soc. 1990, 112, 9013.

(d) Hayashi, N.; Ho, D. M.; Pascal, Jr., R. A. Tetrahedron Lett. 2000, 41, 4261.

(e) Cairns, G. A.; Carr, N.; Green, M.; Mahon, M. F. Chem. Commun. 1996, 2431.

[4] (a) Moriarty, R. M. Penmasta, R.; Awasthi, A. K.; Prakash, I. J. Org. Chem. 1988, 53, 6124.

(b) Sawaki, Y.; Inoue, H.; Ogata, Y. Bull. Chem. Soc. Jpn. 1983, 56, 1133.

(c) Sullivan, B. P.; Smythe, R. S.; Kober, E. M.; Meyer, T. J. J. Am. Chem. Soc. 1982, 104, 4701

[5] Shen, T.; Wang, T.; Qin, C.; Jiao, N.; Angew. Chem., Int. Ed. 2013, $52,6677$.

[6] (a) Wang, A.; Jiang, H. J. Am. Chem. Soc., 2008, 130, 5030. (b) Liu, Q.; Chen, P.; Liu, G. ACS Catal. 2013, 3, 178.

[7] (a) Jun, C.-H.; Lee, H.; Moon, C. W.; Hong, H.-S. J. Am. Chem. Soc. 2001, 123, 8600 .

(b) Cha, K.-M.; Jo, E.-A.; Jun, C.-H. Synlett 2009, 2939.

[8] (a) Das, A.; Chaudhuri, R.; Liu, R.-S. Chem. Commun. 2009, 4046. (b) Liu, Y.; Song, F.; Guo, S. J. Am. Chem. Soc. 2006, $128,11332$. (c) Qin, C.; Su, Y.; Shen, T.; Shi, X.; Jiao, N. Angew. Chem., Int. Ed. 2016, 55, 350 .

[9] Sun, J.; Wang, F.; Hu, H.; Wang, X.; Wu, H.; Liu, Y. J. Org. Chem. 2014, 79, 3992.

[10] (a) Datta, S.; Chang, C.-L.; Yeh, K.-L.; Liu, R.-S. J. Am. Chem. Soc. 2003, 125, 9294. 
(b) Shimada, T.; Yamamoto, Y. J. Am. Chem. Soc. 2003, 125, 6646.

[11] (a) Liang, Y.-F.; Song, S.; Ai, L.; Li, X.; Jiao, N. Green Chem. 2016, 18,6462 .

(b) Sha, H.-K.; Xu, T.; Liu, F.; Tang, B.-Z.; Hao, W.-J.; Tu, S.-J.; Jiang, B. Chem. Commun. 2018, 54, 10415.

(c) Sha, H.-K.; Liu, F.; Lu, J.; Liu, Z.-Q.; Hao, W.-J.; Tang, J.-L.;

Tu, S.-J.; Jiang, B. Green Chem. 2018, 20, 3476.

(d) Wang, L.; Shi, L.-X.; Liu, L.; Li, Z.-X.; Xu, T.; Hao, W.-J.; Li, G.; Tu, S.-J.; Jiang, B. J. Org. Chem. 2017, 82, 3605

[12] (a) Qiu, J.-K.; Jiang, B.; Zhu, Y.-L.; Hao, W.-J.; Wang, D.-C.; Sun, J.; Wei, P.; Tu, S.-J.; Li, G. J. Am. Chem. Soc. 2015, 137, 8928.

(b) Sun, J.; Qiu, J.-K.; Wu, Y.-N.; Hao, W.-J.; Guo, C.; Li, G.; Tu, S.-J.; Jiang, B. Org. Lett. 2017, 19, 754.

(c) Liu, F.; Wang, J.-Y.; Zhou, P.; Li, G.; Hao, W.-J.; Tu, S.-J.; Jiang B., Angew. Chem., Int. Ed. 2017, 56, 15570.

(d) Huang, M.-H.; Hao, W.-J.; Li, G.; Tu, S.-J.; Jiang, B. Chem. Commun. 2018, 54, 10791.

(e) Huang, M.-H.; Hao, W.-J.; Jiang, B. Chem. Asian J. 2018, 13, 2958.

(f) Wang, A.-F.; Hao, W.-J.; Zhu, Y.-L.; Li, G.; Zhou, P.; Tu, S.-J.;
Jiang, B. ACS Omega 2018, 3, 1482.

[13] (a) Wang, J.-Y.; Zhou, P.; Li, G.; Hao W.-J.; Tu, S.-J.; Jiang, B. Org. Lett. 2017, 19, 6682.

(b) Zhou, P.; Wang, J.-Y.; Zhang, T.-S.; Li, G.; Hao, W.-J.; Tu, S.-J.; Jiang, B. Chem. Commun. 2018, 54, 164

(c) Li, H.; Hao, W.-J.; Wang, M.; Qin, X.; Tu, S.-J.; Zhou, P.; Li, G.; Wang, J.; Jiang, B. Org. Lett. 2018, 20, 4362.

[14] For selected examples, see:

(a) Shen, Q.; G. Hammond, B. J. Am. Chem. Soc. 2002, 124, 6534.

(b) Ohno, H.; Mizutani, T.; Kadoh, Y.; Miyamura, K.; Tanaka, T. Angew. Chem., Int. Ed. 2005, 44, 5113.

(c) Siebert, M. R.; Osbourn, J. M.; Brummond, K. M.; Tantillo, D. J. J. Am. Chem. Soc. 2010, 132, 11952.

[15] Li, H.; Hao, W.-J.; Li, G.; Tu, S.-J.; Jiang, B. Front. Chem. 2018, 6, 599.

(b) Li, H.; Zhou, P.; Xie, F.; Hu, J.-Q.; Yang, S.-Z.; Wang, Y.-J.; Hao, W.-J.; Tu, S.-J.; Jiang, B. J. Org. Chem. 2018, 83, 13335.

(c) Wang, J.-Y.; Xie, F.; Hu, J.-Q.; Yang, S.-Z.; Wang, Y.-J.; Hao, W.-J.; Tu, S.-J.; Jiang, B. Org. Biomol. Chem. 2018, 16, 7104.

(Li, L.; Fan, Y.) 